\title{
Association between Preoperative Leukocytosis and Pathological Features of Colorectal Cancers
}

\author{
Reza Hajebi', Hadi Ahmadi Amoli', Hossein Zabihi Mahmoudabadi', Ehsan \\ Rahimpour ${ }^{1}$, Khosro Najjar ${ }^{1}$, Elham Nazar ${ }^{2}$
}

${ }^{1}$ Department of surgery, Sina Hospital, Tehran University of Medical Sciences, Iran. ${ }^{2}$ Department of Pathology, Sina Hospital, Tehran University of Medical Sciences, Iran.

\begin{abstract}
Background: Using hematologic profiles including leukocyte count have been considered as prognostic factor for some cancers. We have been assessed the predictive value of leukocytosis before surgery in colorectal cancers. Methods: This cross-sectional study has been conducted on patients with colorectal cancer who underwent surgery from 2017 to 2019 in Sina hospital, Tehran. The patients were divided in two groups based on high and low leukocyte count before surgery. Results: A total of 84 patients were studied with colorectal cancers including 46 males $(55.4 \%)$ and 37 females $(44.6 \%)$ with the average age of $55.2( \pm 13.63)$ years. $36.1 \%$ of patients had leukocytosis in laboratory data before surgery. There was a significant association between tumor's grade and the incidence of leukocytosis before surgery $(\mathrm{P}<0.001)$. The existence of leukocytosis is highly associated with poorly differentiated tumor. Also, the relationship between the location of the tumor and leukocytosis was seen which the location was distal, the possibility of leukocytosis decreased significantly $(\mathrm{P}<0.005)$. Conclusion: Our findings suggest that the location of the tumor and its degree of tumor differentiation are related with leukocytosis. This current study by considering leukocytosis before surgery aimed to use this variable as a prognostic factor in patients with colorectal cancer alongside with other variables.
\end{abstract}

Keywords: Colorectal cancer- leukocytosis- prognostic factor

Asian Pac J Cancer Biol, 6 (1), 43-47

Submission Date: 01/09/2021 Acceptance Date: 02/28/2021

\section{Introduction}

Colorectal cancer is one of the most common cancers among societies, highly affected by lifestyle. A number of risk factors for colorectal cancer have been recognized such as lack of physical activity, obesity, low use of vegetables and fruits, and smoking [1-6]. Prognostic factors are indicators affecting the overall results of treatment whereas predictive factors are those affecting the response to intervention or any specific treatment [7-8]. Using predictive factors, especially for high risk patients such as those with cancer which require more attention and precise treatments, was considered for appropriate treatment approaches with fewer side effects for patients [9-10]. Changes in blood parameters including anemia, leukopenia, leukocytosis, and thrombocytosis in most malignancies have been well studied and considered as a part of paraneoplastic syndromes.
Paraneoplastic syndromes include non-metastatic systematic manifestations which observe in many malignancies and have negative alter on the prognosis. These non-metastatic manifestations almost always occur in all malignancies, but their patterns in each cancer are different [11]. Many of patients who have abnormality in hematology profile even before the diagnosis is established. Other paraneoplastic syndroms develop during treatment course and related with the progression or regression of the tumor [12-14]. Among paraneoplastic syndromes, leukocytosis (mainly granulocytosis) is more prevalent in non-hematologic malignancies. About 10 percent of patients with non-hematologic malignancies have leukocytosis. This percentage is higher in tumors of lung, head and neck, pancreas, brain, and melanoma. Granulocytosis connect to paraneoplastic leukemoid

Corresponding Author:

Dr. Elham Nazar

Assistant Professor of Pathology, Sina Hospital, Tehran University of Medical Sciences, Iran.

Email: enazar@sina.tums.ac.ir 
reaction which observes with the increased presence of mature neutrophils and some other related forms [15]. Based on recent studies suggest that using hematology profiles including leukocyte count has been considered as prognostic factors in some cancers [16-20]. Hematology profile test is cheap, easy to use, and available in all surgery centers. Particularly during the hospitalization of patients, is suitable to use it [21-22]. In this study, we report the association between preoperative leukocytosis and pathological features of colorectal cancers based on data from the 2017 to 2019 in Sina hospital, Tehran, Iran. The purpose of this study is to investigate prognostic value of leukocytosis before surgery in colorectal cancers.

\section{Materials and Methods}

This is a cross-sectional study included patients with colorectal cancers who were treated in Sina hospital affiliated to Tehran University of Medical Sciences from 2017 to 2019. Inclusion criteria for the study were patients with colorectal cancer (confirmed by pathology result) that underwent surgery with curative goal. Exclusion criteria were the incomplete medical record files, loss of patients during follow up, and patients with abscess, obstruction, and patients who had neoadjuvant chemoradiation's side effects. Recorded variables for each patient were age, sex, tumor location and pathologic characteristic of the tumor after resection (depth of invasion, lymph node status, lymphovascular invasion, perineural invasion, tumor differentiation grade). Laboratory findings were assessed such as Carcinoembryonic antigen (CEA) and complete blood cell count (CBC) by Electrochemiluminescence assay and blood cell analyzer (Sysmex KX21), respectively. Based on the amount of leukocyte (higher or lower than $10000 / \mu 1)$, patients were divided into two groups of leukocytosis and non-leukocytosis, respectively. Other factors including tumor stage, its degree, its anatomical location as well as CEA levels were compared between two groups.

Statistical analysis of the results was performed by using SPSS-16 software (SPSS Inc, Chicago, Illinois, USA). Descriptive and statistical analysis was done. Results were presented as mean \pm standard deviation (SD) for quantitative variables and were summarized by absolute frequencies and percentages for categorical variables. The association between the variables and leukocytosis was examined using by Chi-square test. All statistical tests were 2-sided and P-value less than 0.05 were considered significant.

\section{Results}

The database query returned 179 patients. We excluded 96 patients due to mentioned exclusion criteria. The remaining 83 patients were evaluated which including 46 males $(55.4 \%)$ and 37 females $(44.6 \%)$ with mean age of 55 years and standard deviation of 13.6 years (42-68 years). According to TNM (tumor, node and metastasis system in all its versions) staging: 3 (3.6\%), 17 (20.4\%), $55(66.2 \%)$, and $8(9.6 \%)$ of patients' tumor was in T1, T2,
T3, and T4, respectively. 49 (59\%) patients had no lymph node invasion, but 34 (41\%) had lymph node invasion. Metastasis was present in $3(3.6 \%)$ patients while 80 $(96.4 \%)$ patients had no detectable metastases. Staging of tumor in $14(16.8 \%), 35(42.1 \%), 33(39.7 \%)$, and $3(3.6 \%)$ patients was stage I, stage II, stage III, and stage IV, respectively. Tumor differentiation grading consist of $11(13.2 \%), 57(68.6 \%)$, and $15(18 \%)$ patients with poor, moderate, and well differentiated features, respectively. Location of the tumor in 30 (36.1\%), 5 (6\%), 27 (32.5\%), and $21(25.3 \%)$ patients was in right colon, transverse colon, left colon, and rectum, respectively. Histologic tumor types consist of $85.5 \%$ with classic adenocarcinoma and $14.5 \%$ with mucinous adenocarcinoma, respectively. Mean White blood cell (WBC) count before surgery was $8880( \pm 4051.7) / \mathrm{ml}$, ranging from 2500 to $19800 /$ ml. Mean Hemoglobin (Hbg) level was $11.59( \pm 1.6) \mathrm{gr} /$ $\mathrm{dl}$, ranging from 8.2 to $16 \mathrm{gr} / \mathrm{dl}$. Mean platelet count was $305602( \pm 119267.8) / \mathrm{ml}$, ranging from 12500 to $673000 /$ ml. Increased CEA level was about $17(28.8 \%) \mathrm{ng} / \mathrm{ml}$ in patients.

There was no statistically significant difference in lymphovascular and perineural invasion between the two groups (P-value $>0.05)$. There was no statistically significant difference in tumor staging and presence of metastasis between the two groups ( $\mathrm{P}$-value $>0.05$ ).

There was statistically significant difference in grade of the differentiation tumor between two groups (P-value $<0.05)$. Leukocytosis has been seen in $6.7 \%$ of well differentiated tumors, in $35.1 \%$ of moderately differentiated tumors and in $81.8 \%$ of poorly differentiated tumors. Chi-square test had shown statistically significant correlation between the differentiation of tumor and the existence of leukocytosis $(\mathrm{p}<0.001)$.

Other important finding of the current study was the correlation between leukocytosis and location of the tumor. Data analysis showed that the percentage of tumors accompanied with leukocytosis in right colon cancer and transverse was $60 \%$, in left colon was $22.2 \%$ and in rectum was $14.3 \%$. Chi-square test have shown that presence of leukocytosis had a significant decrease from right colon towards rectum $(\mathrm{p}<0.005)$ (Tables 1$)$.

There was no statistically significant mean between other hematologic findings and CEA level with our variables (Tables 2 ).

\section{Discussion}

Several studies have been conducted on factors affecting the prognosis of colorectal cancers. In these studies, age, sex, location of the tumor, size of the tumor, differentiation degree of the tumor, cancer stage, and the symptoms of disease have been assessed as prognostic factors for colorectal cancer [23]. Several studies regarded hematologic indexes consist of leukocytosis as a predictive factor for prognosis and mortality of the patients which was recognized in majority of solid tumors [21-22]. In a study by Qiu et al. the level of WBC, platelets and hemoglobin in patients with breast, hepatocellular, nasopharyngeal, oropharyngeal, gastric, cervix, endometrial, renal cell, 
Table 1. Tumor Characteristics Based on the Existence or Lack of Leukocytosis

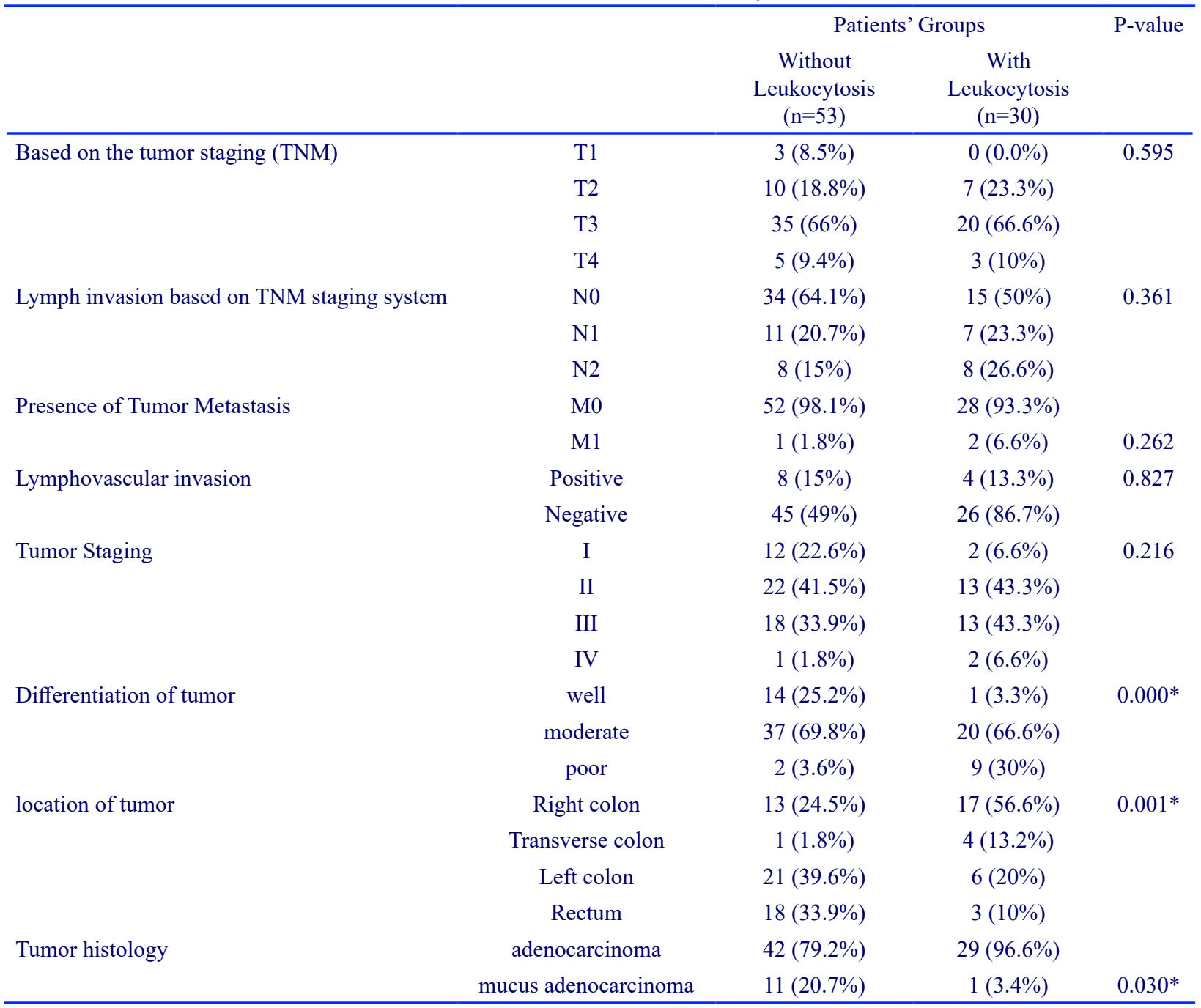

*A p-value $<0.05$ is considered statistically significant.

Table 2. CEA Level Changes in two Groups

\begin{tabular}{lcccc}
\hline & & Without Leukocytosis (34) & With Leukocytosis (25) & P-value \\
\hline CEA changes & Normal & $24(70.5 \%)$ & $18(72 \%)$ & 0.906 \\
& Increased & $10(29.5 \%)$ & $7(28 \%)$ & \\
\hline
\end{tabular}

and lung cancers was assessed which showed only leukocytosis can be an independent prognostic factor in patients with cervix cancer [22]. Studies showed that patients with cervix cancer who had leukocytosis (WBC count $>10,000 / \mathrm{ml}$ ), had lower treatment success and survival comparing to those without leukocytosis. Moreover, these patients had a higher activity of immune system comparing to groups without leukocytosis. Leukocytosis before surgery along with other variables such as the stage of disease, tumor diameter, and histology grade of tumor are the main prognostic factors in the patients and can be used for determination of the patients with poor prognosis [11]. In a study by Banerjee et al. it had been shown that leukocytosis in prior treatment of anal cancer was associated with lower life expectancy and survival. It seems that hematologic indexes can be used as the potential prognostic factors. This study showed that leukocytosis in the patients related with decreased two- year survival and disease free survival. Also, the patients with accompanying anemia and leukocytosis had more decreased survival than the patients with only leukocytosis [17]. The current study demonstrates that leukocytosis is related to the location of tumor and degree of tumor differentiation which associated with poor prognosis. In a study by Nakshabendi et al. was conducted on patients with colorectal cancer which showed WBC to Hbg ratio was high and considered as concurrency of anemia $(\mathrm{Hbg}<10 \mathrm{gr} / \mathrm{dl})$ and leukocytosis. The ratio was higher in patients with right colon cancer about $55 \%$ while in those with left colon cancer was about $10 \%$ which the difference was statistically significant. Such studies had shown that patients with right colon cancer were more prone to develop leukocytosis and anemia [19] which is similar to our findings. Qiu et al. study established that anemia and thrombocytosis before treatment were independent prognostic factors for patients'survival in advanced 
stages of the disease. In addition, the synergistic effect of anemia and thrombocytosis decreased the survival of the patients more than each one alone [21]. Several studies compared leukocytosis, anemia, and thrombocytosis between patients with colorectal cancers and patients with benign colorectal lesions including benign colorectal polyps. These results showed hematologic indexes were significantly higher among patients with colorectal cancers, especially in advanced stages of the disease [23]. The current study by considering leukocytosis before surgery aimed to use this variable as a prognostic factor in the patients with colorectal cancer alongside with other variables. Our results show that the leukocytosis is related to the location and degree of tumor differentiation. Right colon cancer has more prominent leukocytosis than the other site and poorly differentiated tumor has more leukocytosis than well and moderately differentiated tumor. Unfortunately, our study has some limitations including limited sample size and we couldn't differentiate WBCs because all samples hadn't differential count. Also, for the reason that large disparities exist between population in cancer incidence, sign, and survival, further studies in Iran with larger scale in several main surgery center is recommended.

In conclusion, based on current study, the location and differentiation degree of the tumor is associated with the existence or lack of leukocytosis. The existence of leukocytosis is highly associated with tumor in the right colon as well as poor differentiation degree of tumor. So, we can use CBC test as prognostic factor easily.

\section{Conflict of Interest}

None declared

\section{References}

1. Parkin DM, Bray F, Ferlay J, Pisani P. Global Cancer Statistics, 2002. CA: A Cancer Journal for Clinicians. 2005 03 01;55(2):74-108. https://doi.org/10.3322/canjclin.55.2.74

2. Parkin DM. International variation. Oncogene. 2004 08;23(38):6329-6340. https://doi.org/10.1038/ sj.onc. 1207726

3. Center MM, Jemal A, Ward E. International Trends in Colorectal Cancer Incidence Rates. Cancer Epidemiology Biomarkers \& Prevention. 2009 06;18(6):1688-1694. https:// doi.org/10.1158/1055-9965.epi-09-0090

4. Giovannucci E, Wu K. Cancers of the colon and rectum. Cancer epidemiology and prevention. 2006;3:829.

5. Botteri E, Iodice S, Bagnardi V, Raimondi S, Lowenfels $\mathrm{AB}$, Maisonneuve P. Smoking and Colorectal Cancer. JAMA. 2008 Dec 17;300(23):2765. https://doi.org/10.1001/ jama.2008.839

6. Giovannucci E. Modifiable risk factors for colon cancer. Gastroenterology Clinics of North America. 2002 Dec;31(4):925-943. https://doi.org/10.1016/s08898553(02)00057-2

7. Popkin BM. The Nutrition Transition: An Overview of World Patterns of Change. Nutrition Reviews. 2004 07;62:S140-S143. https://doi.org/10.1111/j.1753-4887.2004. tb00084.x

8. Van Belle SJ, Cocquyt V. Impact of haemoglobin levels on the outcome of cancers treated with chemotherapy. Critical
Reviews in Oncology/Hematology. 2003 07;47(1):1-11. https://doi.org/10.1016/s1040-8428(03)00093-3

9. Sengeløv L, Kamby C, Geertsen P, Andersen LJ, von der Maase H. Predictive factors of response to cisplatin-based chemotherapy and the relation of response to survival in patients with metastatic urothelial cancer. Cancer Chemotherapy and Pharmacology. 2000 Nov 06;46(5):357364. https://doi.org/10.1007/s002800000176

10. Keating MJ, Smith TL, Lerner S, O'brien S, Robertson LE, Kantarjian H, Freireich EJ. Prediction of Prognosis Following Fludarabine Used as Secondary Therapy for Chronic Lymphocytic Leukemia. Leukemia \& Lymphoma. 2000 01;37(1-2):71-85. https://doi. org/10.3109/10428190009057630

11. Garcia-Arias A, Cetina L, Candelaria M, Robles E, DueñasGonzález A. The prognostic significance of leukocytosis in cervical cancer. International Journal of Gynecologic Cancer. 2007;17(2):465-470. https://doi.org/10.1111/j.15251438.2007.00816.x

12. Littlewood TJ, Bajetta E, Nortier JW, Vercammen E, Rapoport B, . Effects of Epoetin Alfa on Hematologic Parameters and Quality of Life in Cancer Patients Receiving Nonplatinum Chemotherapy: Results of a Randomized, Double-Blind, Placebo-Controlled Trial. Journal of Clinical Oncology. 200106 01;19(11):2865-2874. https://doi. org/10.1200/jco.2001.19.11.2865

13. Granger JM, Kontoyiannis DP. Etiology and outcome of extreme leukocytosis in 758 nonhematologic cancer patients. Cancer. 200909 01;115(17):3919-3923. https:// doi.org/10.1002/cncr.24480

14. Sakka V, Tsiodras S, Giamarellos-Bourboulis EJ, Giamarellou H. An update on the etiology and diagnostic evaluation of a leukemoid reaction. European Journal of Internal Medicine. 2006 Oct;17(6):394-398. https://doi. org/10.1016/j.ejim.2006.04.004

15. Hiraki A, Ueoka H, Takata I, Gemba K, Bessho A, Segawa Y, Kiura K, Eguchi K, Yoneda T, Tanimoto M, Harada M. Hypercalcemia-leukocytosis syndrome associated with lung cancer. Lung Cancer. 2004 03;43(3):301-307. https://doi. org/10.1016/j.lungcan.2003.09.006

16. Kasuga I, Makino S, Kiyokawa H, Katoh H, Ebihara Y, Ohyashiki K. Tumor-related leukocytosis is linked with poor prognosis in patients with lung carcinoma. Cancer. 2001;92(9):2399-2405. https://doi.org/10.1002/10970142(20011101)92:9<2399::aid-cncr1588>3.0.co;2-w

17. Banerjee R, Roxin G, Eliasziw M, Joseph K, Buie D, Doll C. The prognostic signficance of pretreatment leukocytosis in patients with anal cancer treated with radical chemoradiotherapy or radiotherapy. Journal of Clinical Oncology. 2012;30(4_suppl):656.

18. Mabuchi S, Matsumoto Y, Isohashi F, Yoshioka Y, Ohashi H, Morii E, Hamasaki T, Aozasa K, Mutch DG, Kimura T. Pretreatment leukocytosis is an indicator of poor prognosis in patients with cervical cancer. Gynecologic Oncology. 2011 07;122(1):25-32. https://doi.org/10.1016/j. ygyno.2011.03.037

19. Nakashabendi I, Dawodu J, Lee F, Russell R, Mikhail M, Dragenscu J. The predictive value of concurrent anaemia and leukocytosis in the diagnosis of colorectal cancer. Gastroenterology. 1998;114:A652.

20. Tomita M, Shimizu T, Hara M, Ayabe T, Onitsuka T. Preoperative leukocytosis, anemia and thrombocytosis are associated with poor survival in non-small cell lung cancer. Anticancer research. 2009;29(7):2687-90.

21. Qiu M, Yuan Z, Luo H, Ruan D, Wang Z, Wang F, Li Y, Xu R. Impact of pretreatment hematologic profile on survival 
of colorectal cancer patients. Tumor Biology. 201003 25;31(4):255-260. https://doi.org/10.1007/s13277-0100024-x

22. Qiu M, Xu R, Ruan D, Li Z, Luo H, Teng K, Wang Z, Li Y, Jiang W. Incidence of anemia, leukocytosis, and thrombocytosis in patients with solid tumors in China. Tumor Biology. 201007 23;31(6):633-641. https://doi.org/10.1007/ s13277-010-0079-8

23. Zuli Y, Jianping W, Lei W, Wenguang D, Yihua H, Jianzhang $\mathrm{Q}$, et al. Multivariate regression analysis of prognostic factors in colorectal cancer. The Chinese-German Journal of Clinical Oncology. 2003;2(3):149-52.

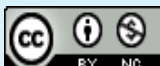

This work is licensed under a Creative Commons AttributionNon Commercial 4.0 International License. 